GSA Data Repository 2016064

\title{
Isotopic evidence for iron mobility during subduction
}

\author{
B. Debret ${ }^{1,2 *}$, M.-A. Millet ${ }^{1}$, M.-L. Pons ${ }^{1,2}$, P. Bouilhol ${ }^{1}$, E. Inglis ${ }^{1}$, and H. Williams ${ }^{1,2}$ \\ ${ }^{1}$ Department of Earth Sciences, Durham University, Durham DH1 3LE, U.K. \\ ${ }^{2}$ Department of Earth Sciences, The University of Cambridge, Downing Street, Cambridge CB2 \\ 3EQ, U.K. \\ *ba.debret@gmail.com
}

\section{GEOLOGICAL SETTING}

Our study focuses on the Piemont zone of the south-western Alps where ophiolitic units were metamorphosed and juxtaposed during alpine subduction and collision in Late Cretaceous to Tertiary (e.g. Tricart, 1984). We have sampled a serpentinite suite from 5 meta-ophiolites that record metamorphic conditions ranging from greenschist to eclogite facies (see Debret et al., 2014 for more detailed about this sample suite). The Chenaillet ophiolite is located in the external Piemont zone, $6 \mathrm{~km}$ west of Briançon (Fig. DR1). It is a thin tectonic nappe resting upon the Queyras Schistes Lustrés complex (Caby, 1995). It is composed of metagabbroic pods and serpentinites recording greenschist facies conditions (Mevel et al., 1978). The Queyras Schistes Lustrés complex is composed of metamorphosed Mesozoic oceanic sediments, strongly deformed during alpine subduction (Tricart and Schwartz, 2006). The complex encloses boudinaged ophiolite units composed of serpentinites, metagabbros and metabasalts from meter to kilometer size, and records blueschist facies P-T conditions (Schwartz et al., 2013). The Monte Maggiore ophiolite is located in the northern end of the Cap Corse, Northern Corsica (Fig. DR1). It is an ultramafic body of $\sim 4 \mathrm{~km}^{2}$ surrounded by eclogitized continental units. The massif is composed of slightly serpentinized peridotites, serpentinites and metagabbros recording blueschist facies conditions (e.g. Vitale-Brovarone et al., 2013). The Monviso meta-ophiolite is located in the extreme East of the Queyras Schistes Lustrés complex (Fig. DR1). This ultramafic body is composed of kilometric lenses of metabasites embedded in highly deformed serpentinites recording eclogite facies P-T conditions (Schwartz et al., 2001). The Lanzo massif is an eclogitized ultramafic body of $150 \mathrm{~km} 2$ bounded by the sediments of the Po plain to the East and South, meta-ophiolites and the Schistes Lustrés unit in the West, and by the HP metamorphic continental unit of Sesia in the North (Pelletier and Müntener, 2006).

\section{MAJOR ELEMENTS AND Fe $\mathrm{Fe}^{3+} / \mathbf{2 F}$ DETERMINATION}

Major element concentrations were determined by ICP-AES at the Laboratoire Magmas et Volcans of Clermont-Ferrand (France) following procedures described in Debret et al. (2014a). Concentrations were calibrated against the DR-N basaltic glass (Govindaraju, 1994). Precision on samples were determined by repeated analyses of the reference basalt BHVO. The reproducibility is better than $5 \%$ for all measured elements. Additional $\mathrm{Fe}^{2+}$ analyses of bulk rock samples were performed at the SARM-CRPG (Nancy, France). These analyses were done by automatic titration at the equivalent point with potassium dichromate after dissolution of the sample in a $\mathrm{HF} / \mathrm{H}_{2} \mathrm{SO}_{4}$ mixture, in the presence of $\mathrm{H}_{3} \mathrm{BO}_{3}$ and $\mathrm{H}_{3} \mathrm{PO}_{4}$ 
(Andreani et al., 2013). Subsequently $\mathrm{Fe}^{3+} / \Sigma \mathrm{Fe}$ ratios were calculated from the measured $\mathrm{Fe}_{2} \mathrm{O}_{3 \text { total }}$ and $\mathrm{Fe}^{2+}$ Total values (e.g. Andreani et al., 2013). The method has been tested by Debret et al. (2015), who were able to match measured bulk $\mathrm{Fe}^{3+} / \Sigma \mathrm{Fe}$ ratios with those recalculated from $\mu$-XANES measurements of serpentine minerals (see Debret et al., 2014 and 2015 for more details on the method including standard ran in the XANES sessions, calibration and precision).

\section{TRACE ELEMENTS}

Trace element concentrations ( $\mathrm{Li}, \mathrm{Sc}, \mathrm{Ti}, \mathrm{V}, \mathrm{Co}, \mathrm{Ni}, \mathrm{Cu}, \mathrm{Zn}, \mathrm{As}, \mathrm{Rb}, \mathrm{Sr}, \mathrm{Y}, \mathrm{Zr}, \mathrm{Nb}, \mathrm{Cd}, \mathrm{Sb}, \mathrm{Cs}, \mathrm{Ba}$, Rare Earth Elements (REE), Hf, Ta, Pb, Th, and U) are from Debret et al. (2013), Lafay et al. (2013) and this study (Table DR2). In this study, they were analysed at the National Oceanography Centre, Southampton using a Thermo X-Series Quadrupole ICP-MS. $10 \mathrm{mg}$ of powdered sample were digested by addition of concentrated hydrofluoric and nitric acids. These solutions were then dried down and the reside dissolved in $2 \% \mathrm{HNO}_{3}$, which was spiked with $10 \mathrm{ng} / \mathrm{mL}$ In and Re for use as an internal correction. The external precision and accuracy of the analyses were assessed by measuring as unknown three rock standards: BHVO and BIR-1 basalts and JA-2 peridotite. Our results show good agreement between measured values and expected values for international standards, and external reproducibility is within $0-5 \%$ for $\mathrm{Sc}$, Ti, V, Ni, Cu, As, Rb, Sr, Y, Cd, Sb, Ba, La, Ce, Nd, Sm, Eu, Gd, Tb, Ho, Tm, Lu, Li, Co, Pr, Dy, Er, and $\mathrm{Yb}$ and within $10-30 \%$ for $\mathrm{Cs}, \mathrm{Hf}, \mathrm{Nb}, \mathrm{Ta}, \mathrm{Pb}, \mathrm{Th}, \mathrm{U}$ and $\mathrm{Zr}$.

The Western Alps serpentinites have relatively uniform major element compositions that overlap with those of abyssal peridotites (Bodinier and Godard, 2013). Rare earth element (REE) concentration patterns of Western Alps SSP and Liz-serpentinite vary according to rock type, and range from lherzoliteto dunitic- like patterns. The lherzolite like pattern is characterized by depletion in light rare earth elements $\left(\mathrm{Ce}_{\mathrm{N}} / \mathrm{Yb}_{\mathrm{N}} \sim 0.1 ; \mathrm{N}\right.$ : $\mathrm{C} 1$-chondrites normalized; LREE) and a flat medium and heavy rare earth element (M-HREE) segment $\left(\mathrm{Gd}_{\mathrm{N}} / \mathrm{Yb}_{\mathrm{N}} \sim 1\right)$. The dunite-like pattern is concave and characterized by an enrichment in HREE relative to MREE $\left(\mathrm{Gd}_{\mathrm{N}} / \mathrm{Yb}_{\mathrm{N}} \sim 0.6\right)$. The slightly serpentinized peridotites (SSP) display both positive and negative Eu anomalies, which probably reflect melt/rock interaction processes (Bodinier and Godard, 2013). Compared to the SSP, the Liz- and Atg/Liz- serpentinites can display enrichment in LREE relative to MREE and/or a negative anomaly in Ce relative to neighbouring elements reflecting seawater/rock interactions (Rouméjon et al., 2015) and the highest LREE mobility relative to M-HREE during sea floor serpentinization process (Paulick et al., 2006). During subduction, the prograde crystallization of lizardite and antigorite do not fractionate REE composition significantly, such that even highly metamorphosed serpentinites retain their protolith REE patterns (Debret et al., 2013). Atg- and Atg/Ol- serpentinites display similar patterns to lherzolites, which are characterized by depletion in LREE

relative to M-HREE with the exception of two samples (Vis1F and Vis5b) which display U-shaped patterns.

\section{Fe ISOTOPE ANALYSES}

Iron isotope analyses were performed by multiple-collector inductively coupled plasma mass spectrometry (MC-ICPMS; Thermo Neptune Plus) at Durham University, utilizing a ${ }^{57} \mathrm{Fe}-{ }^{58} \mathrm{Fe}$ double spike (Millet et al., 2012) and/or by simple standard bracketing (Williams et al., 2005, Hibbert et al., 2012, Williams and Bizimis, 2014; Table DR1) to correct for instrumental mass bias. Both methods give 
results within analytical error (Table DR1, Fig. DR3). Dissolution, iron purification and isotopic analyses were undertaken at Durham University using established procedures (Hibbert et al., 2012, Williams and Bizimis, 2014). In the case of double-spike analyses, solutions consisted of a mixture of $1.5 \mathrm{ppm} \mathrm{Fe}$ of $0.75 \mathrm{ppm}$ sample and $0.75 \mathrm{ppm}$ double spike in $0.1 \mathrm{M} \mathrm{HNO}_{3}$, whereas for sample-standard bracketing they consisted of $2 \mathrm{ppm}$ natural $\mathrm{Fe}$ in $0.1 \mathrm{M} \mathrm{HNO}_{3}$. The standard Fe beam intensities typically varied between 25 and $35 \mathrm{~V}^{56} \mathrm{Fe}$ for a standard $10^{-11} \Omega$ resistor. Mass dependence, long-term reproducibility and accuracy were evaluated by analysis of an in-house $\mathrm{FeCl}$ salt standard $\left(\delta^{56} \mathrm{Fe}=-0.71 \pm 0.05 \%\right.$; $\delta^{57} \mathrm{Fe}=$ $-1.05 \pm 0.09 \%$ o $2 \mathrm{sd}, n=67$ ) previously analysed in other studies (Williams et al., 2005, 2014; Weyer and Ionov, 2007; Millet et al., 2012). The USGS standards BIR-1 (Icelandic basalt) were also analysed over the course of this study. The mean Fe isotope composition of this standard is: $\delta^{56} \mathrm{Fe}=0.05 \pm 0.06 \%$; $\delta^{57} \mathrm{Fe}=0.07 \pm 0.05 \%$ o $(2 \mathrm{sd}, n=4)$. 


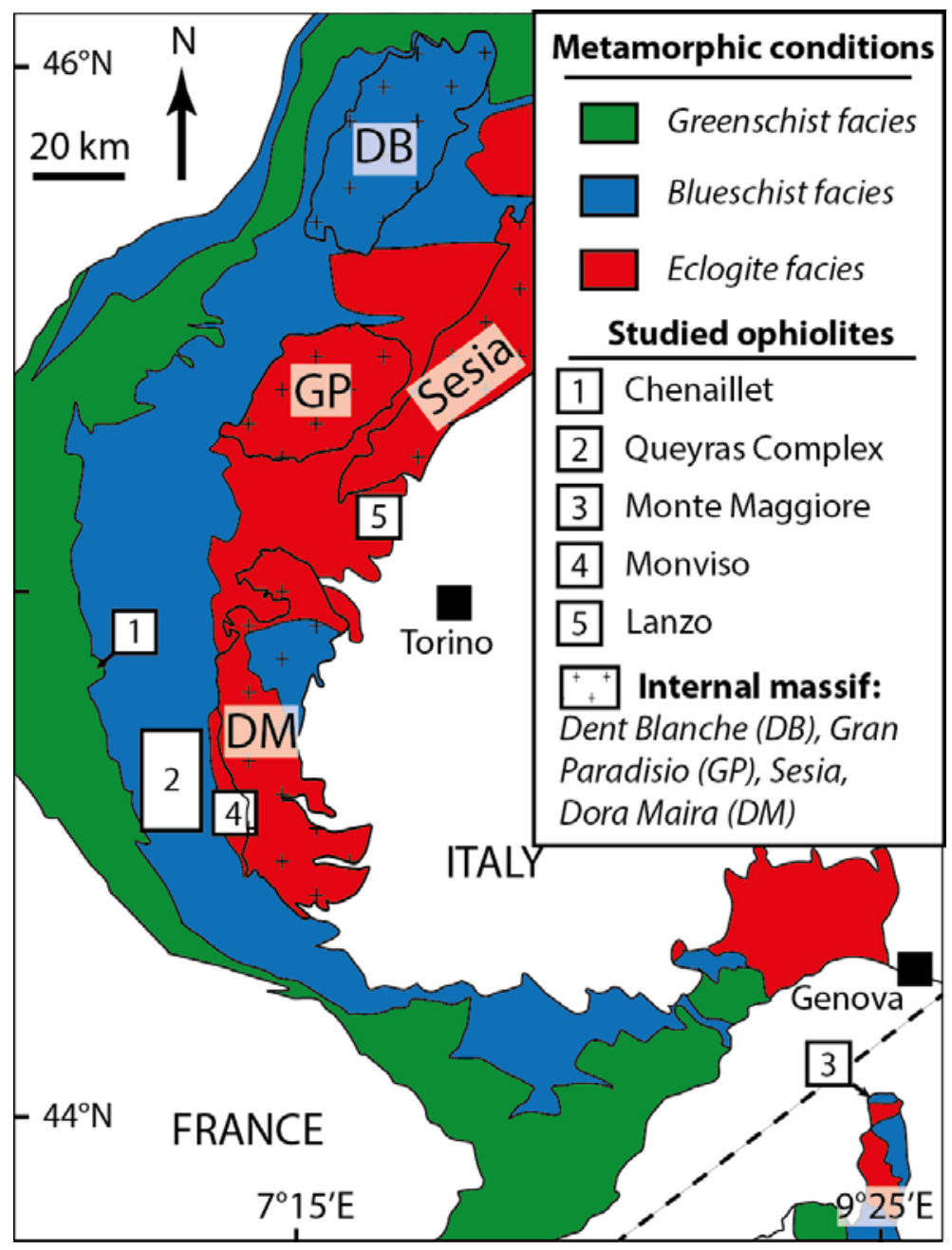

Figure DR1. Simplified metamorphic map of the Western Alps showing the spatial distribution of the studied ultramafic ophiolites (numbered white squares). 


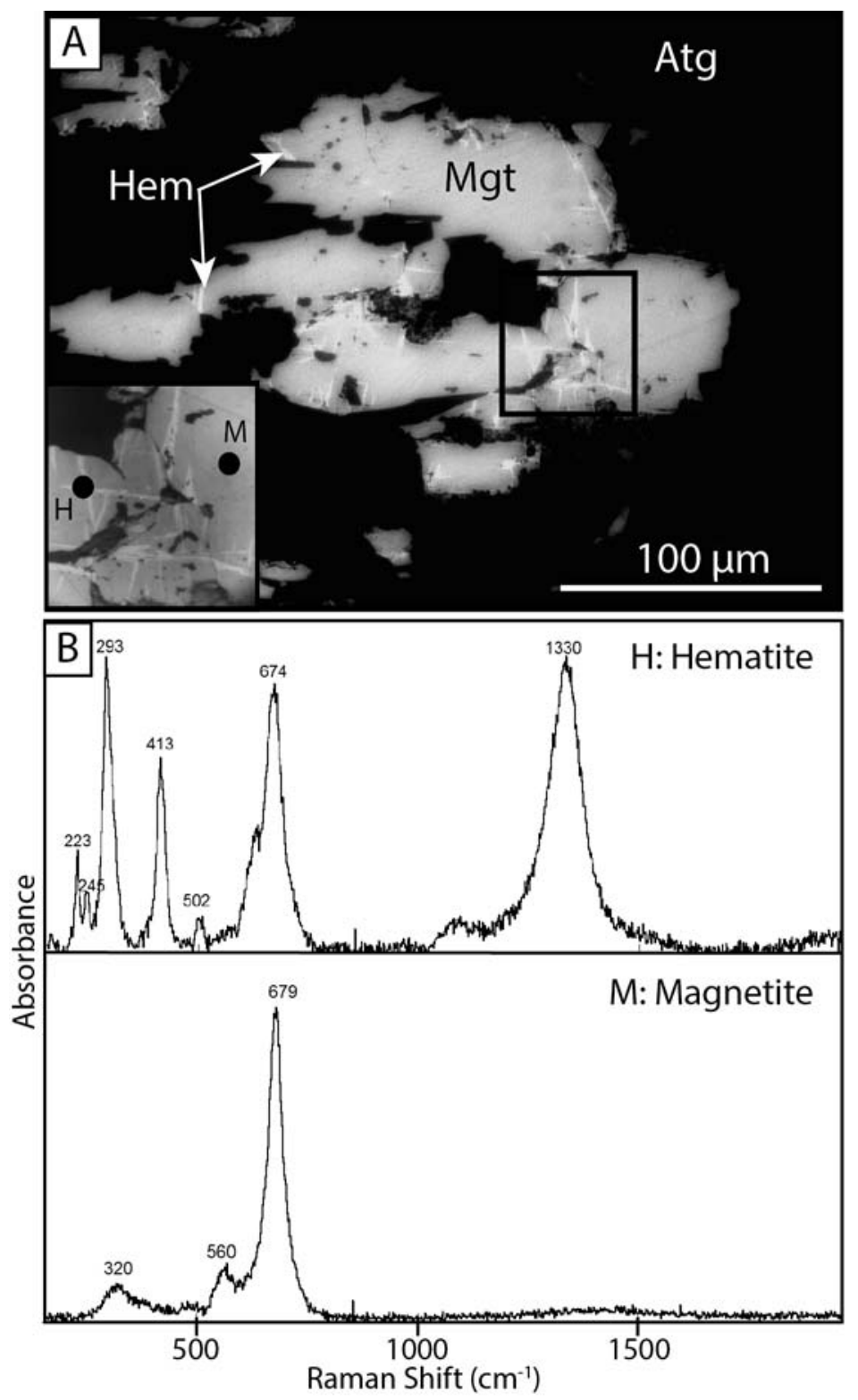

Figure DR2. A microphotograph of an Atg-serpentinite displaying magnetite grains containing white needles of hematite. The box displays a zoom of hematite needles with location of Raman analyses. Raman analyses of the white needles are characterized by four peaks at 223, 293, 413, and $1330 \mathrm{~cm}^{-1}$ corresponding to hematite. The Raman spectrum of magnetite is characterized by three peaks at 320, 560 and $679 \mathrm{~cm}^{-1}$. 


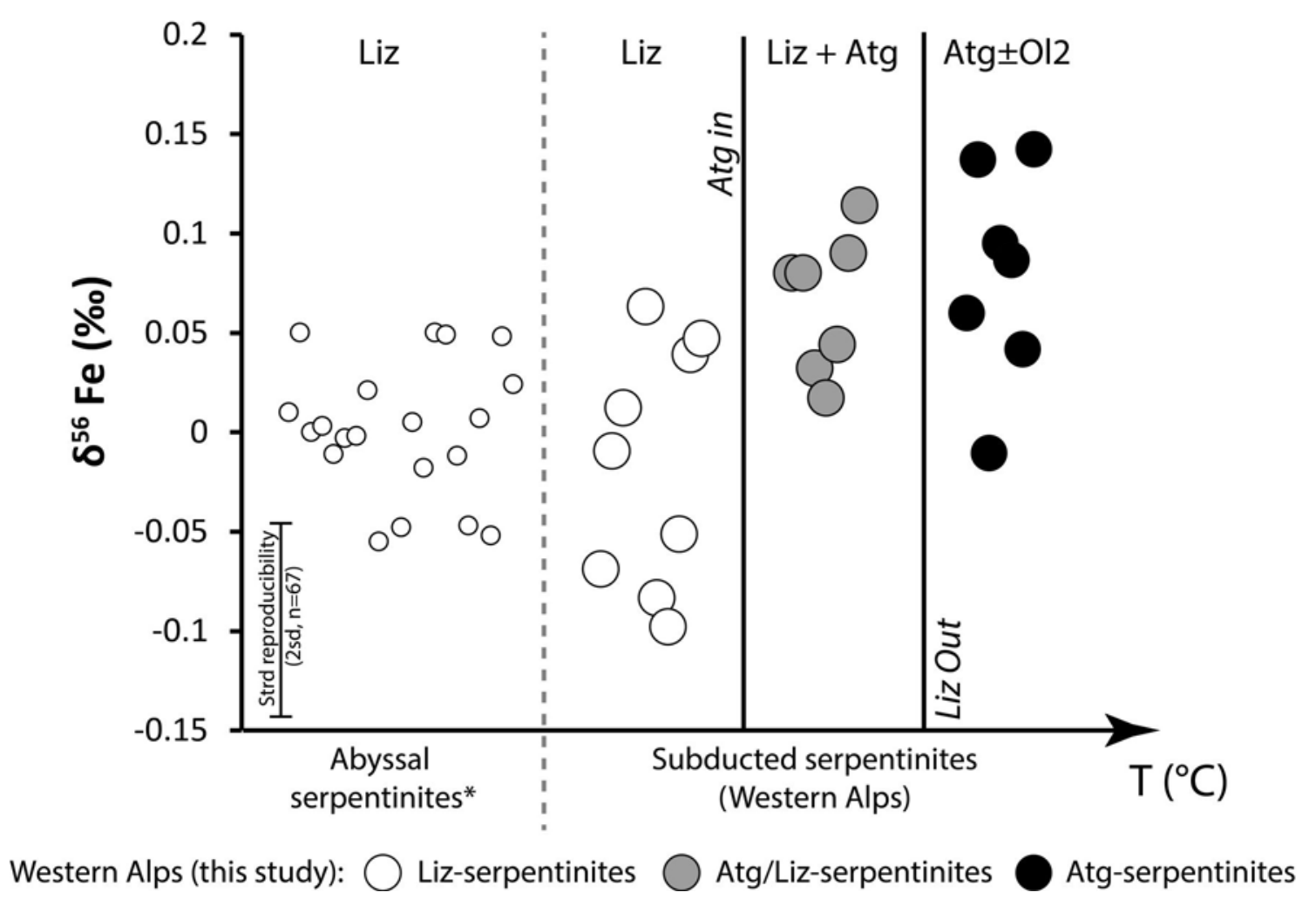

Figure DR3. Iron isotope composition of abyssal serpentinites (South West Indian Ridge and Gakkel ridge) and serpentinites from Western Alps ophiolites. Abyssal serpentinite values are from Craddock et al. (2013, non-weathered rocks). T-test indicates that the Liz-serpentinites and Atg/Liz-serpentinites are significantly different from each other at the $90 \%$ confidence interval $(t=-3.05$, d.f. $=14)$. 

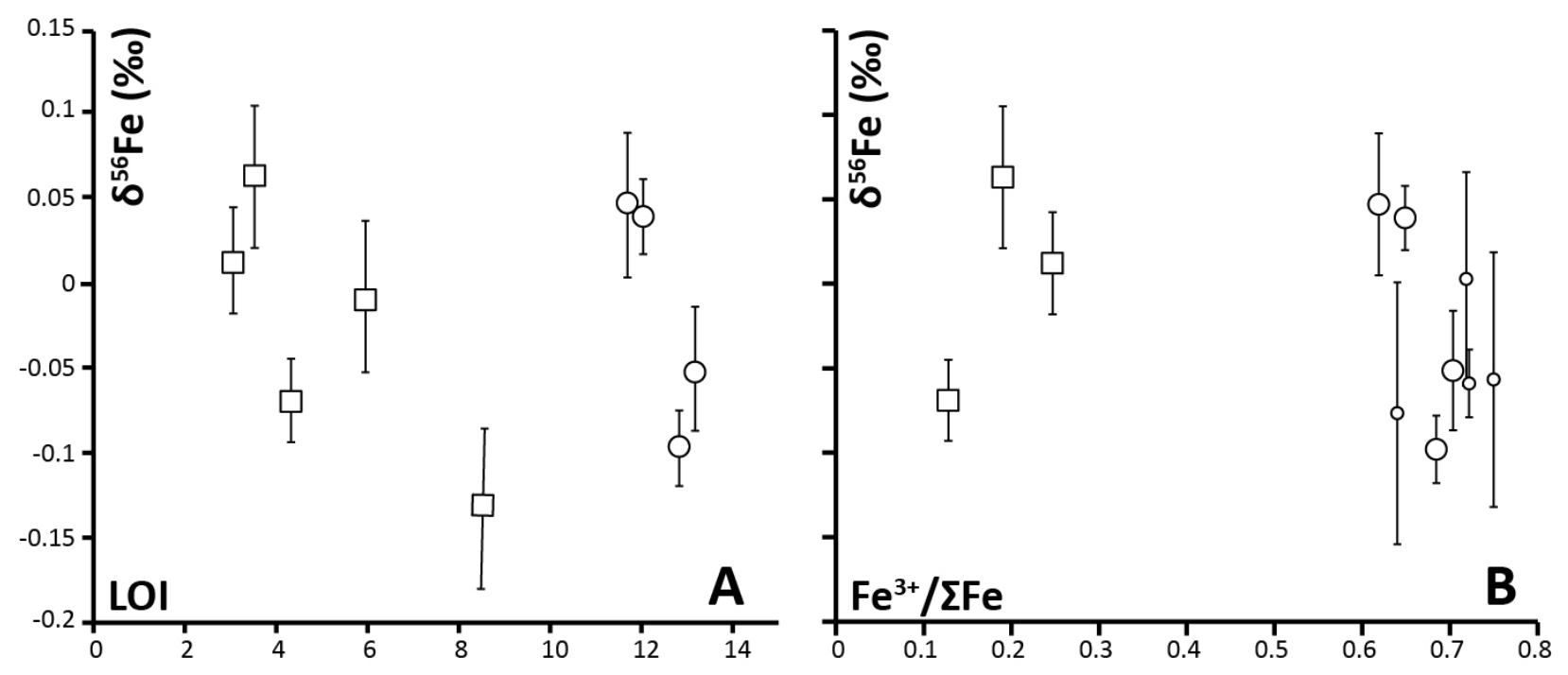

Figure DR4. Evolution of $\delta^{56} \mathrm{Fe}$ as a function of peridotite serpentinization degree. Iron isotope composition $\left(\delta^{56} \mathrm{Fe}\right)$ of alpine SSP (white squares) and Liz-serpentinites (white circles) versus serpentinization degree proxies (A: Loss on ignition; $\mathrm{B}: \mathrm{Fe}^{3+} / \Sigma \mathrm{Fe}$ ). Small circles correspond to abyssal serpentinites (Gakkel Ridge samples). Both plots show that there is no variation of iron isotopes in peridotite during serpentinization process. 


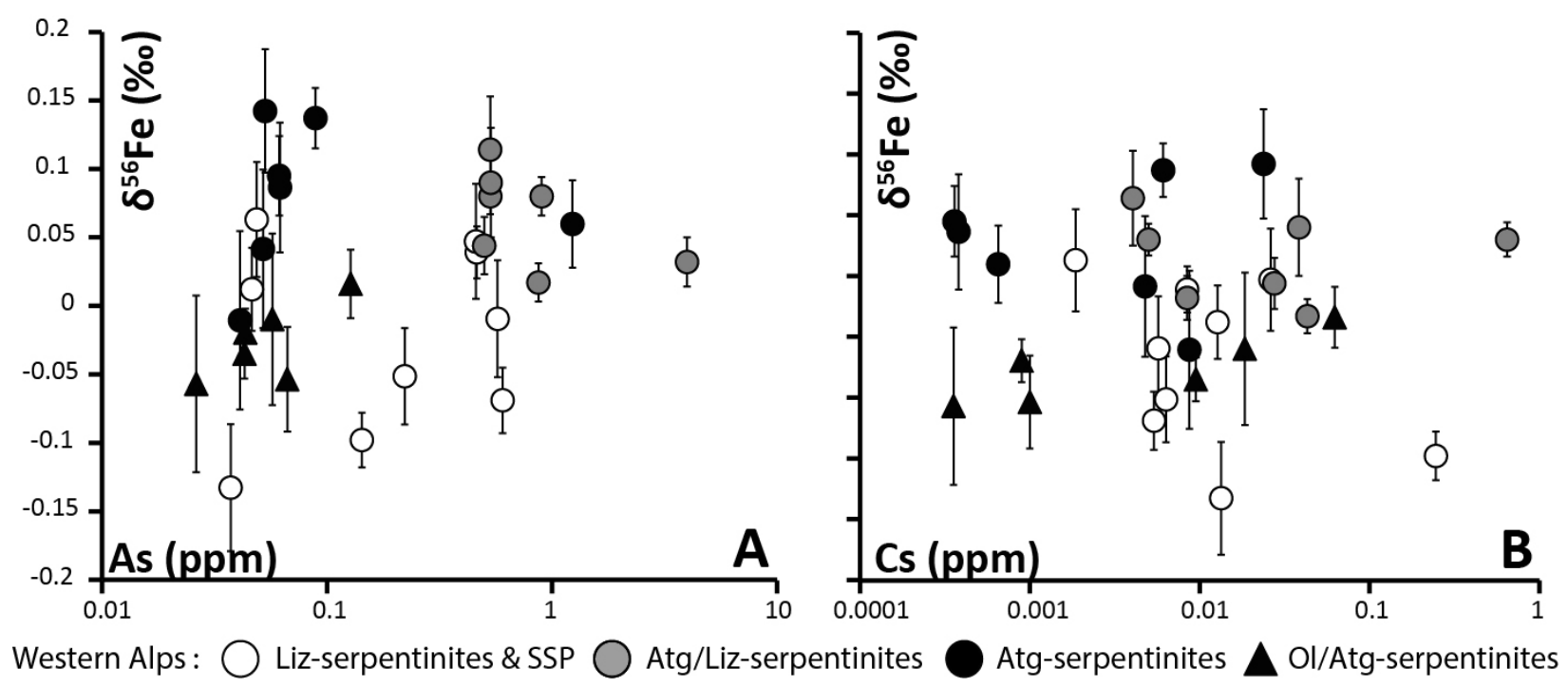

Figure DR5. Plot of $\delta^{56} \mathrm{Fe}$ versus elemental tracers of sediment/serpentinite interactions. Both plots show that there is no correlation between both parameters showing that addition or release of $\mathrm{Fe}$ by sediment/serpentinite interactions is unlikely. 


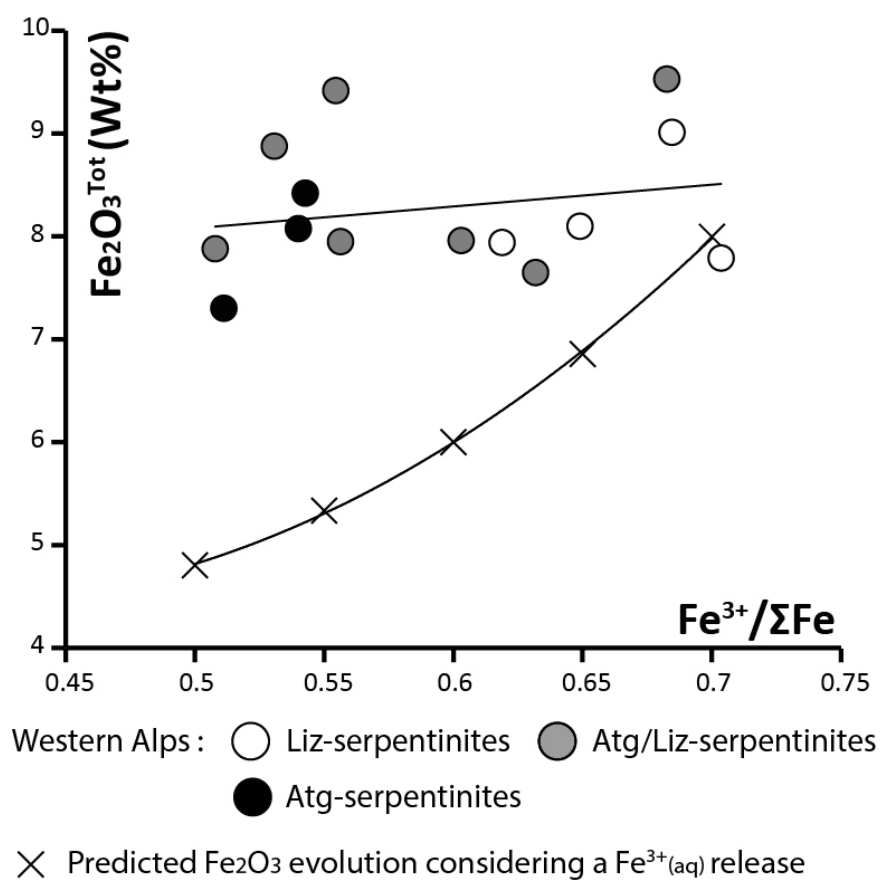

Figure DR6: Plot of $\mathrm{Fe}_{2} \mathrm{O}_{3 \text { total }}$ and $\mathrm{Fe}^{3+} / \Sigma \mathrm{Fe}$ in alpine serpentinites. There is no obvious change of the $\mathrm{Fe}_{2} \mathrm{O}_{3 \text { total }}$ in serpentinites during prograde metamorphism. At the opposite, the release of $\mathrm{Fe}^{3+}$ requires a decrease of about $3 \mathrm{wt} \%$ of $\mathrm{Fe}_{2} \mathrm{O}_{3}$ in fluids to decrease the $\mathrm{Fe}^{3+} / \Sigma \mathrm{Fe}$ from 0.7 to 0.5 . 


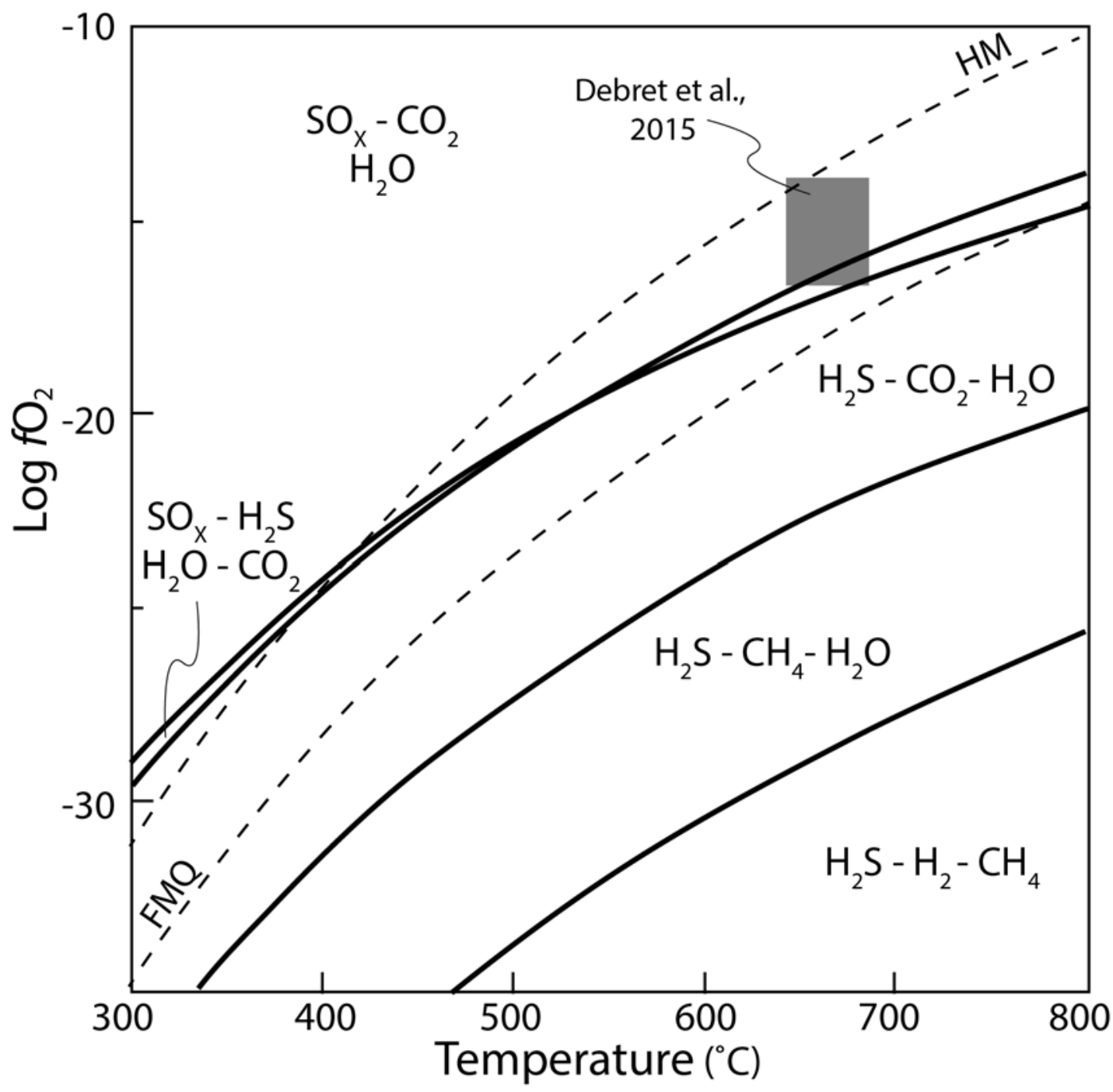

Figure DR7. $\mathrm{Log} \mathrm{fO}_{2}$-T diagram illustrating the redox conditions of $\mathrm{H}-\mathrm{S}-\mathrm{C}-\mathrm{O}$ fluids at $1.5 \mathrm{GPa}$ (modified after Ohmoto, 1986). The dotted lines show the $\mathrm{fO}_{2}-\mathrm{T}$ conditions of well-known mineral buffers (HM: hematite-magnetite; FMQ: Fayalite-Magnetite-Quartz). The grey box shows the $\mathrm{fO}_{2}-\mathrm{T}$ conditions of metaperidotite crystallization during subduction (Debret et al., 2015). Sulfates are presented under $\mathrm{SO}_{\mathrm{X}}$ form since the disproportionation of $\mathrm{SO}_{2}$ can contribute to form $\mathrm{SO}, \mathrm{SO}_{3}{ }^{2-}, \mathrm{S}_{2} \mathrm{O}_{3}{ }^{2-}$ or $\mathrm{SO}_{4}{ }^{2-}$ rich fluids (e.g. Gamo et al., 1997). 
Table DR1: Iron isotope composition of studied ultramafic rocks

\begin{tabular}{|c|c|c|c|c|c|c|c|c|c|c|c|c|}
\hline Sple & $\begin{array}{c}\text { Serp. } \\
\text { Mineral }\end{array}$ & $\begin{array}{c}\mathrm{Fe}_{2} \mathrm{O}_{3 \text { total }} \\
(\mathrm{wt} \%)\end{array}$ & $\mathrm{Fe}^{3+} / \Sigma \mathrm{Fe}$ & $\mathrm{Al}_{2} \mathrm{O}_{3} / \mathrm{SiO}_{2}$ & $\begin{array}{l}\delta^{56} \mathrm{Fe} \\
(\%))^{*}\end{array}$ & 2 sd & $\mathbf{N}$ & $\begin{array}{c}\delta^{56} \mathrm{Fe} \\
(\%)\end{array}$ & $2 s d$ & $\begin{array}{c}\delta^{57} \mathrm{Fe} \\
(\% \mathrm{o}) \\
\end{array}$ & $2 \mathrm{sd}$ & $\mathbf{N}$ \\
\hline \multicolumn{13}{|c|}{ Sligthly Serpentinized peridotite } \\
\hline Mag26 & - & 8.3 & 0.13 & 0.05 & -0.069 & $\begin{array}{l}0.0 \\
24\end{array}$ & 2 & -0.100 & - & -0.171 & - & 1 \\
\hline Mag33 & - & 8.4 & - & 0.06 & -0.010 & $\begin{array}{l}0.0 \\
43\end{array}$ & 1 & 0.020 & $\begin{array}{c}0.03 \\
9\end{array}$ & 0.047 & $\begin{array}{c}0.10 \\
7\end{array}$ & 4 \\
\hline LZ17a & - & 8.9 & 0.25 & 0.06 & 0.012 & $\begin{array}{l}0.0 \\
30\end{array}$ & 2 & -0.020 & - & -0.037 & - & 1 \\
\hline LZ19 & - & 8.9 & 0.19 & 0.07 & 0.063 & $\begin{array}{l}0.0 \\
42\end{array}$ & 4 & 0.035 & $\begin{array}{c}0.03 \\
7\end{array}$ & 0.029 & $\begin{array}{c}0.03 \\
4\end{array}$ & 2 \\
\hline LZ7 & - & 6.8 & - & 0.01 & - & - & & -0.133 & $\begin{array}{c}0.04 \\
6\end{array}$ & -0.213 & $\begin{array}{c}0.10 \\
8\end{array}$ & 3 \\
\hline \multicolumn{13}{|l|}{ Liz-Serpentinite } \\
\hline $\begin{array}{l}\text { HLY102D707 } \\
3\end{array}$ & Liz & $7.6^{* *}$ & 0.64 & - & - & - & & -0.077 & $\begin{array}{c}0.07 \\
8\end{array}$ & -0.109 & $\begin{array}{c}0.13 \\
2\end{array}$ & 4 \\
\hline $\begin{array}{l}\text { HLY102D709 } \\
9\end{array}$ & $\mathrm{Liz}$ & $7.5^{* *}$ & 0.72 & - & -0.059 & $\begin{array}{l}0.0 \\
20\end{array}$ & 2 & - & - & - & - & - \\
\hline $\begin{array}{l}\text { HLY102D870 } \\
8\end{array}$ & $\mathrm{Liz}$ & $4.7 * *$ & 0.86 & - & - & - & & -0.057 & $\begin{array}{c}0.07 \\
5\end{array}$ & -0.125 & $\begin{array}{c}0.19 \\
9\end{array}$ & 2 \\
\hline PS5924619 & Liz & $6.7^{* *}$ & 0.72 & - & - & - & & 0.003 & $\begin{array}{c}0.06 \\
3\end{array}$ & 0.036 & $\begin{array}{c}0.13 \\
9\end{array}$ & 8 \\
\hline BCh10 & $\mathrm{Liz}$ & 8.1 & 0.65 & 0.05 & 0.039 & $\begin{array}{c}0.0 \\
19\end{array}$ & 6 & 0.034 & $\begin{array}{c}0.01 \\
1\end{array}$ & 0.045 & $\begin{array}{c}0.05 \\
3\end{array}$ & 2 \\
\hline ICh02 & Liz & 7.8 & 0.70 & 0.02 & -0.051 & $\begin{array}{l}0.0 \\
35\end{array}$ & 2 & -0.062 & $\begin{array}{c}0.07 \\
8\end{array}$ & -0.096 & $\begin{array}{c}0.02 \\
8\end{array}$ & 2 \\
\hline BCh6 & $\operatorname{Liz} \pm \mathrm{Atg}$ & 7.9 & 0.62 & 0.07 & 0.047 & $\begin{array}{l}0.0 \\
42\end{array}$ & 4 & 0.006 & $\begin{array}{c}0.07 \\
0\end{array}$ & 0.003 & $\begin{array}{c}0.10 \\
2\end{array}$ & 2 \\
\hline RQ30 & $\mathrm{Liz} \pm \mathrm{Atg}$ & 9.0 & 0.68 & 0.04 & -0.098 & $\begin{array}{l}0.0 \\
20\end{array}$ & 3 & - & - & - & - & - \\
\hline \multicolumn{13}{|c|}{ Atg/Liz-serpentinite } \\
\hline BCH9 & Liz/Atg & 7.9 & 0.56 & 0.08 & 0.080 & $\begin{array}{c}0.0 \\
13\end{array}$ & 6 & 0.034 & - & 0.059 & - & 1 \\
\hline RQ23 & Liz/Atg & 9.4 & 0.55 & 0.02 & 0.080 & $\begin{array}{c}0.0 \\
14\end{array}$ & 6 & - & - & - & - & - \\
\hline RQ01 & $\operatorname{Atg} \pm \operatorname{Liz}$ & 7.6 & 0.63 & 0.03 & 0.032 & $\begin{array}{c}0.0 \\
18\end{array}$ & 6 & - & - & - & - & - \\
\hline MM8 & Liz/Atg & 9.5 & 0.68 & 0.05 & 0.044 & $\begin{array}{l}0.0 \\
21\end{array}$ & 3 & & & & & 2 \\
\hline MM15 & Liz/Atg & 7.9 & 0.51 & 0.05 & 0.114 & $\begin{array}{l}0.0 \\
39\end{array}$ & 4 & - & - & - & - & - \\
\hline MM19 & $\operatorname{Atg} \pm \operatorname{Liz}$ & 8.9 & 0.53 & 0.05 & 0.090 & $\begin{array}{c}0.0 \\
40\end{array}$ & 3 & 0.115 & $\begin{array}{c}0.09 \\
5\end{array}$ & 0.125 & $\begin{array}{c}0.06 \\
4\end{array}$ & 2 \\
\hline MM2 & Atg & 8.0 & 0.60 & 0.05 & 0.017 & $\begin{array}{r}0.0 \\
14\end{array}$ & 6 & 0.006 & $\begin{array}{c}0.01 \\
2\end{array}$ & -0.014 & $\begin{array}{c}0.01 \\
4\end{array}$ & 2 \\
\hline \multicolumn{13}{|l|}{ Atg-serpentinite } \\
\hline LZ30 & Atg & 6.2 & - & 0.06 & - & - & & 0.060 & $\begin{array}{c}0.03 \\
2\end{array}$ & 0.126 & $\begin{array}{c}0.08 \\
5\end{array}$ & 3 \\
\hline LZ14b & Atg & 8.4 & 0.54 & 0.06 & 0.137 & $\begin{array}{l}0.0 \\
22\end{array}$ & 2 & 0.117 & $\begin{array}{c}0.03 \\
4\end{array}$ & 0.188 & $\begin{array}{c}0.01 \\
7\end{array}$ & 2 \\
\hline
\end{tabular}




\begin{tabular}{|c|c|c|c|c|c|c|c|c|c|c|c|c|}
\hline LZ8b & Atg & 9.7 & - & 0.06 & - & - & & -0.011 & $\begin{array}{c}0.06 \\
5\end{array}$ & -0.002 & $\begin{array}{c}0.09 \\
6\end{array}$ & 5 \\
\hline LZ34d & Atg & 7.3 & 0.51 & 0.08 & - & - & & 0.142 & $\begin{array}{c}0.04 \\
5\end{array}$ & 0.210 & $\begin{array}{c}0.10 \\
0\end{array}$ & 4 \\
\hline Vis1 & Atg & 8.1 & 0.54 & 0.05 & 0.095 & $\begin{array}{l}0.0 \\
29\end{array}$ & 2 & 0.129 & $\begin{array}{c}0.07 \\
2\end{array}$ & 0.174 & $\begin{array}{c}0.21 \\
5\end{array}$ & 2 \\
\hline Vis1F & Atg & 6.1 & - & 0.04 & - & - & & 0.086 & $\begin{array}{c}0.04 \\
7\end{array}$ & 0.133 & $\begin{array}{c}0.03 \\
1\end{array}$ & 4 \\
\hline Vis9 & Atg & 9.2 & - & 0.04 & - & - & & 0.042 & $\begin{array}{c}0.05 \\
8\end{array}$ & 0.082 & $\begin{array}{c}0.09 \\
0\end{array}$ & 6 \\
\hline \multicolumn{13}{|c|}{ Atg/Ol2-serpentinite } \\
\hline LZ5 & Atg & 8.1 & - & 0.05 & - & - & & -0.010 & $\begin{array}{c}0.06 \\
3\end{array}$ & -0.009 & $\begin{array}{c}0.11 \\
1\end{array}$ & 4 \\
\hline LZ26a & Atg & 5.6 & - & 0.06 & - & - & & -0.020 & $\begin{array}{c}0.01 \\
8\end{array}$ & -0.043 & $\begin{array}{c}0.05 \\
3\end{array}$ & 2 \\
\hline RO1 & Atg & 8.1 & 0.53 & 0.07 & 0.016 & $\begin{array}{l}0.0 \\
25\end{array}$ & 2 & -0.003 & - & 0.023 & - & 1 \\
\hline LZ27a & Atg & 8.5 & 0.24 & 0.06 & -0.035 & $\begin{array}{c}0.0 \\
18\end{array}$ & 6 & - & - & - & - & - \\
\hline Vis12 & Atg & 7.5 & - & 0.03 & - & - & & -0.054 & $\begin{array}{c}0.03 \\
8\end{array}$ & -0.082 & $\begin{array}{c}0.05 \\
0\end{array}$ & 4 \\
\hline Vis $5 \mathrm{~b}$ & Atg & 9.2 & 0.52 & 0.03 & - & - & & -0.057 & $\begin{array}{c}0.06 \\
5\end{array}$ & -0.130 & $\begin{array}{c}0.14 \\
2\end{array}$ & 4 \\
\hline
\end{tabular}

*Double spike values **determined by MC-ICP-MS 
Table DR2: Compilation of trace element (ppm) analyses

\begin{tabular}{|c|c|c|c|c|c|c|c|c|c|c|c|c|c|c|c|c|c|}
\hline & Iag26 & ag33 & Z17a & Z35b & LZ19 & LZ7 & ICH02 & BCh10 & BCh6 & Ch9 & IM2 & MM8 & MM19 & MM15 & LZ26c & $\mathbf{Z 3 0}$ & ZZ14b \\
\hline $\mathrm{Li}$ & 62 & 05 & 2.83 & .10 & 80 & 51 & 265.65 & 51 & 1.50 & & & 0.55 & 0.86 & 59 & 0.08 & 01 & 0.00 \\
\hline $\mathrm{Sc}$ & 2.51 & 39 & 0 & .37 & 75 & 09 & n.d. & 10 & & .34 & & 11.10 & .01 & 11.60 & 2.48 & 1.58 & 11.43 \\
\hline $\mathrm{V}$ & 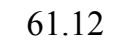 & & & 72.08 & & & $\mathrm{n}$. & & & & & & & & & & 56.83 \\
\hline $\mathrm{Cr}$ & 50 & 08 & d. & n.d. & n.d. & n.d. & n.d. & 0 & 24 & 39 & 19 & 991 & 742 & 2648 & n.d. & n.d. & n.d. \\
\hline Co & 94.48 & 87.02 & 99.24 & 83.19 & 91.00 & 81.24 & 93.55 & 82.82 & & 4.88 & & & & & 23 & & 79.81 \\
\hline $\mathrm{Ni}$ & 28 & 706 & 09 & 2 & 4 & 5 & & & 2 & 17 & & 1604 & 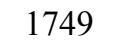 & & 1201 & & 1821 \\
\hline $\mathrm{Cu}$ & 15.550 & 20.760 & 21.414 & 31.382 & 18.096 & 0.499 & 27.966 & 6.700 & 1.139 & 6.444 & 11.740 & 7.376 & 2.529 & 12.010 & 6.179 & 2.785 & 1.574 \\
\hline $\mathrm{Zn}$ & 46.020 & 40.940 & 42.820 & 43.493 & 47.981 & 3.711 & 146.297 & 33.270 & 3.690 & 35.330 & 37.440 & 32.250 & 31.250 & 51. & 38.103 & 43.304 & 28.871 \\
\hline As & 0.603 & 572 & 46 & 057 & 0,040 & 037 & 221 & 463 & 458 & & 0.869 & & & & 34 & .231 & 0.089 \\
\hline $\mathrm{Rb}$ & 0.040 & 9 & 0. & 0 & 0.059 & 0. & & 0. & & & & & & & 0.005 & 2 & 0.051 \\
\hline $\mathrm{Sr}$ & 0.685 & & 1.282 & 19.158 & 5.436 & 0.999 & 41 & & 28 & & & & & & 27 & & 0.286 \\
\hline Y & 1.734 & 593 & 2. & 3 & 3. & 0.131 & 0 & 1. & 1 & & 1 & 94 & 9 & & 1.126 & 3 & 0.593 \\
\hline $\mathrm{Zr}$ & 0.723 & 490 & 04 & 797 & 4.800 & 10 & 0.693 & 0.678 & 279 & 2.258 & 0.321 & 0.361 & 3.298 & 0.449 & 0.759 & 0.354 & 0.875 \\
\hline $\mathrm{Nb}$ & 0 & & & & & & & & & & & & & & & & 17 \\
\hline $\mathrm{Sb}$ & 0.012 & , & 0.006 & 0.004 & 0. & 0.004 & 0.028 & 0.004 & 0.006 & 7 & 0. & 0.020 & 0.014 & & 0.007 & 0.110 & 0.015 \\
\hline Cs & 0.005 & & 0.013 & 2 & 0.002 & 0.013 & 0.006 & 0.008 & 0.026 & 0.005 & & & 8 & & 000 & .001 & 0.006 \\
\hline $\mathrm{Ba}$ & 0 & & & & & & & & & & & & & & & & 0.185 \\
\hline $\mathrm{La}$ & 0.011 & 1 & 07 & 0.169 & 997 & 0.016 & 0.058 & 0.003 & 0.016 & 0.057 & 0.2 & 0.008 & 0.529 & 0.007 & 0.005 & 0.004 & 0.053 \\
\hline $\mathrm{Ce}$ & 6 & & & & & & & & & & & & & & 19 & 06 & 110 \\
\hline $\operatorname{Pr}$ & 0 & & 0.020 & & & 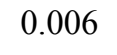 & & 0.011 & 0.039 & & & & & & 5 & 0.002 & 0.028 \\
\hline $\mathrm{Nd}$ & 0.077 & & 200 & 0.855 & 0.601 & 0.037 & 0 & 09 & 32 & 5 & 0.3 & 0.092 & 696 & & .044 & .013 & 0.165 \\
\hline $\mathrm{Sm}$ & 0.067 & & & & & & & & & & & & & & & & 0.063 \\
\hline $\mathrm{Eu}$ & 0.033 & & 57. & 4 & 10 & 0007 & 0 & 0.035 & 0.058 & 79 & 0.0 & 3 & 0.096 & 8 & 0.009 & 0.007 & 0.009 \\
\hline $\mathrm{Gd}$ & & & & & & & 0.056 & 44 & 4 & & 0.1 & 0 & 0.287 & 0.1 & 0.059 & 0.024 & \\
\hline $\mathrm{Tb}$ & & & & & & & & & & & & & & & & & 0.019 \\
\hline Dy & 0.280 & 56 & 88 & 0.7 & 0.616 & 0.021 & 0.055 & 0.229 & 33 & 0.442 & 0.219 & 61 & 0.391 & 0 & 0.113 & .050 & 0.136 \\
\hline Ho & 0.068 & & & & & & & & & & & & & & 0.032 & 0.015 & 0.029 \\
\hline Er & 0.216 & & 0256 & & & & & 0177 & & 0.295 & & & & & 0.124 & 0.060 & 0.081 \\
\hline $\mathrm{Tm}$ & 0.035 & & & & & & & & & & 2 & 29 & & & & & 0.013 \\
\hline $\mathrm{Yb}$ & & & & & & & & & & & & & & & 0.182 & 0.111 & 0.088 \\
\hline $\mathrm{Lu}$ & 0.039 & & 0.046 & 0.072 & 0.062 & 0.006 & & & & & & & & & 0.035 & 0.022 & 0.015 \\
\hline $\mathrm{Hf}$ & 0.049 & & & & & & & & & & 0.025 & & & 0.036 & 0.046 & 0.030 & 0.040 \\
\hline $\mathrm{Ta}$ & b.d.l. & b.d.l. & 0.0004 & 0.0037 & 0.0013 & 0.0007 & 0.0009 & b.d.l. & b.d.l. & b.d.l. & b.d.l. & b.d.l. & b.d.l. & b.d.l. & 0.0002 & 0.0002 & 0.0003 \\
\hline $\mathrm{Pb}$ & 0.134 & 0.124 & b.d.l. & b.d.l. & 0.060 & b.d.l. & 4.988 & 0.138 & 0.173 & 0.214 & 0.180 & 0.291 & 0.923 & 0.115 & b.d.l. & 0.112 & 0.141 \\
\hline
\end{tabular}




\begin{tabular}{|c|c|c|c|c|c|c|c|c|c|c|c|c|c|c|c|c|c|}
\hline Th & 0.005 & 0.005 & 0.001 & 0.005 & 0.001 & 0.001 & 0.011 & 0.001 & 0.002 & 0.011 & 0.001 & 0.002 & 0 . & 0.001 & b.d.l. & 0.001 & 0.002 \\
\hline 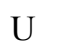 & 0.0019 & 0.0177 & 0.0004 & 0.0018 & 0.0004 & 0.0007 & 0.0088 & 0.0004 & .0009 & 0.0021 & .0020 & 0.0005 & 0.0406 & 0.0032 & 0.0003 & 0.0004 & 60 \\
\hline
\end{tabular}

n.d.: non detected, b.d.1.: below detection limit; data are from Debret et al. (2013), Lafay et al. (2013) and this study 


\begin{tabular}{|c|c|c|c|c|c|c|c|c|c|c|c|c|c|}
\hline LZ8b & LZ5 & LZ26a & RO1 & LZ27A & LZ34d & Vis1 & Vis1F & Vis9 & Vis12 & Vis5b & RQ30 & RQ23 & RQ01 \\
\hline 0.05 & 2.00 & 0.03 & 0.16 & 1.14 & 0.45 & 0.14 & 0.01 & 0.07 & 0.19 & 0.02 & 2109.91 & 483.39 & 49.51 \\
\hline 9.18 & 8.90 & 11.23 & 12.68 & 7.87 & 10.65 & 9.85 & 12.15 & 5.82 & 12.13 & 6.89 & n.d. & n.d. & n.d. \\
\hline 58.61 & 47.02 & 50.87 & 65.65 & 37.48 & 57.29 & 59.68 & 62.93 & 31.79 & 48.10 & 27.57 & n.d. & n.d. & n.d. \\
\hline n.d. & n.d. & n.d. & n.d. & n.d. & n.d. & n.d. & n.d. & n.d. & n.d. & n.d. & n.d. & n.d. & n.d. \\
\hline 93.93 & 95.77 & 76.13 & 97.86 & 97.28 & 85.77 & 94.11 & 80.68 & 55.28 & 95.19 & 61.96 & 93.56 & 98.05 & 82.89 \\
\hline 1283 & 2245 & 1154 & 2246 & 2126 & 2106 & 2266 & 1910 & 1143 & 1943 & 1212 & 1819 & 2021 & 1083 \\
\hline 11.577 & 11.290 & 7.492 & 9.372 & 4.116 & 6.342 & 13.873 & 7.374 & 3.580 & 6.771 & 4.468 & 14.448 & 43.427 & 6.560 \\
\hline 47.145 & 38.504 & 37.225 & 50.157 & 35.278 & 36.180 & 29.457 & 37.150 & 24.331 & 39.427 & 22.231 & 37.000 & 56.700 & 44.856 \\
\hline 0.041 & 0.057 & 0.043 & 0.127 & 0.043 & 0.053 & 0.061 & 0.062 & 0.052 & 0.067 & 0.026 & 0.143 & 0.898 & 3.974 \\
\hline 0.029 & 0.056 & 0.017 & 0.104 & 0.055 & 0.077 & 0.011 & 0.013 & 0.021 & 0.010 & 0.003 & 0.154 & 0.199 & 0.069 \\
\hline 0.537 & 6.083 & 0.152 & 1.821 & 7.411 & 9.986 & 0.530 & 0.146 & 0.174 & 2.516 & 0.223 & 4.606 & 2.040 & 0.297 \\
\hline 1.522 & 1.896 & 1.203 & 2.455 & 1.711 & 3.008 & 1.822 & 1.055 & 0.347 & 0.982 & 0.260 & 1.302 & 0.571 & 0.494 \\
\hline 2.006 & 3.456 & 0.980 & 2.629 & 2.621 & 4.470 & 1.041 & 0.062 & 0.066 & 0.700 & 0.204 & 0.404 & 1.248 & 0.074 \\
\hline 0.016 & 0.003 & 0.006 & 0.027 & 0.016 & 0.027 & 0.009 & 0.004 & 0.013 & 0.007 & 0.005 & 0.011 & 0.023 & 0.012 \\
\hline 0.008 & 0.010 & 0.004 & 0.012 & 0.004 & 0.008 & 0.007 & 0.009 & b.d.l. & 0.010 & 0.014 & 0.014 & 0.129 & 0.995 \\
\hline 0.009 & 0.018 & 0.001 & 0.062 & 0.009 & 0.024 & 0.000 & 0.000 & 0.005 & 0.001 & 0.000 & 0.246 & 0.647 & 0.008 \\
\hline 0.804 & 0.724 & b.d.l. & 8.886 & 0.204 & 0.364 & 0.223 & 0.135 & 0.331 & 0.695 & b.d.l. & 3.024 & 1.085 & 0.895 \\
\hline 0.025 & 0.055 & 0.028 & 0.050 & 0.086 & 0.127 & 0.047 & 0.019 & 0.013 & 0.088 & 0.014 & 0.036 & 0.103 & 0.056 \\
\hline 0.098 & 0.255 & 0.089 & 0.201 & 0.340 & 0.500 & 0.124 & 0.024 & 0.043 & 0.290 & 0.027 & 0.127 & 0.208 & 0.130 \\
\hline 0.021 & 0.056 & 0.016 & 0.053 & 0.064 & 0.099 & 0.023 & 0.003 & 0.007 & 0.045 & 0.003 & 0.025 & 0.028 & 0.014 \\
\hline 0.151 & 0.369 & 0.109 & 0.390 & 0.392 & 0.641 & 0.152 & 0.017 & 0.045 & 0.230 & 0.016 & 0.157 & 0.134 & 0.063 \\
\hline 0.079 & 0.157 & 0.052 & 0.186 & 0.153 & 0.256 & 0.072 & 0.013 & 0.016 & 0.078 & 0.008 & 0.062 & 0.038 & 0.016 \\
\hline 0.020 & 0.062 & 0.002 & 0.091 & 0.075 & 0.105 & 0.009 & 0.003 & 0.003 & 0.025 & 0.003 & 0.026 & 0.026 & 0.008 \\
\hline 0.175 & 0.292 & 0.123 & 0.353 & 0.249 & 0.437 & 0.165 & 0.051 & 0.035 & 0.125 & 0.022 & 0.116 & 0.056 & 0.035 \\
\hline 0.036 & 0.051 & 0.024 & 0.064 & 0.044 & 0.077 & 0.032 & 0.014 & 0.007 & 0.023 & 0.005 & 0.023 & 0.010 & 0.008 \\
\hline 0.296 & 0.379 & 0.194 & 0.484 & 0.325 & 0.566 & 0.274 & 0.142 & 0.054 & 0.176 & 0.041 & 0.175 & 0.073 & 0.069 \\
\hline 0.068 & 0.082 & 0.046 & 0.105 & 0.071 & 0.121 & 0.065 & 0.039 & 0.014 & 0.040 & 0.010 & 0.041 & 0.017 & 0.017 \\
\hline 0.212 & 0.228 & 0.136 & 0.303 & 0.207 & 0.340 & 0.194 & 0.135 & 0.047 & 0.121 & 0.036 & 0.125 & 0.054 & 0.056 \\
\hline 0.034 & 0.035 & 0.022 & 0.044 & 0.031 & 0.051 & 0.029 & 0.024 & 0.008 & 0.018 & 0.007 & 0.019 & 0.009 & 0.009 \\
\hline 0.225 & 0.221 & 0.150 & 0.286 & 0.208 & 0.330 & 0.173 & 0.171 & 0.060 & 0.121 & 0.053 & 0.132 & 0.074 & 0.071 \\
\hline 0.039 & 0.039 & 0.026 & 0.049 & 0.036 & 0.057 & 0.026 & 0.030 & 0.011 & 0.021 & 0.011 & 0.025 & 0.015 & 0.012 \\
\hline 0.074 & 0.108 & 0.043 & 0.108 & 0.083 & 0.145 & 0.028 & 0.008 & 0.004 & 0.026 & 0.007 & 0.019 & 0.039 & 0.006 \\
\hline 0.0012 & 0.0007 & 0.0004 & 0.0019 & 0.0013 & 0.0028 & 0.0006 & 0.0001 & 0.0007 & 0.0005 & 0.0003 & 0.0008 & 0.0018 & 0.0009 \\
\hline b.d.l. & b.d.l. & b.d.l. & 0.139 & b.d.l. & b.d.l. & 0.090 & 0.063 & b.d.l. & 0.067 & b.d.l. & b.d.l. & 1.541 & 1.071 \\
\hline
\end{tabular}




\begin{tabular}{cccccccccccccc}
0.002 & 0.001 & 0.001 & 0.004 & 0.002 & 0.003 & 0.003 & 0.001 & 0.001 & 0.003 & 0.001 & 0.001 & 0.005 & 0.014 \\
0.0007 & 0.0014 & 0.0008 & 0.0011 & 0.0009 & 0.0010 & 0.0008 & 0.0006 & 0.0009 & 0.0012 & 0.0003 & b.d.l. & 0.0036 & 0.0030 \\
\hline
\end{tabular}




\section{REFERENCES CITED:}

Andreani, M., Muñoz, M., Marcaillou, C., and Delacour, A., 2013, $\mu$ XANES study of iron redox state in serpentine during oceanic serpentinization: Lithos, v. 178, p. 70-83.

Bodinier, J.L., and Godard, M., 2013, Orogenic, Ophiolitic, and Abyssal Peridotites, in R.W. Carlson, ed., Mantle and Core: Treatise on Geochemistry, v. 2, p. 103-151.

Caby, R., 1995, Plastic deformations of gabbros in a slow-spreading mesozoic Ridges: example of the Montgenèvre Ophiolite, Western Alps. In: Vissers, R.L.M., Nicolas, A. (Eds.), Proc. Workshop on Mantle and Lower Crust Exposed in Oceanic Ridges and in Ophiolites. Kluwer, Dordrecht, pp. 123-145.

Debret, B., Andreani, M., Godard, M., Nicollet, C., Schwartz, S., and Lafay, R., 2013, Trace element behavior during serpentinization/deserpentinization of an eclogitized oceanic lithosphere: a LAICPMS study of the Lanzo ultramafic massif (Western Alps): Chemical Geology, v. 357, p. 117133.

Debret, B., Andreani, M., Muñoz, M., Bolfan-Casanova, N., Carlut, J., Nicollet, C., Schwartz, S., and Trcera, N., 2014, Evolution of Fe redox state in serpentine during subduction: Earth and Planetary Science Letters, v. 400, p. 206-218.

Debret, B., Bolfan-Casanova, N., Padrón-Navarta, J.A., Martin-Hernandez, F., Andreani, M., Garrido, C., López Sanchez-Vizcaino, V., Gomez-Pugnaire, M.T., Muñoz, M., and Trcera, N., 2015, Redox state of iron during high pressure serpentinite dehydration: Contribution to Mineralogy and Petrology, v. 169:36.

Gamo, T., Okamura, K., Charlou, J.L., Urabe, T., Auzende, J.M., Ishibashi, J., Shitashima, K. and H. Chiba, 1997, Acidic and sulfate-rich hydrothermal fluids from the Manus back-arc basin, Papua New Guinea: Geology, v. 25 , p. 139-142.

Govindaraju, K., 1994, Compilation of working values and sample description for 383 geostandards: Geostandards Newsletter, v. 18, p. 1-158.

Hibbert, K.E.J., Williams, H.M., Kerr, A.C., Puchtel, I.S., 2012. Iron isotopes in an-cient and modern komatiites: evidence in support of an oxidised mantle from Archean to present: Earth and Planetary Science Letters, v. 321, p. 198-207.

Lafay, R., Deschamps, F., Schwartz, S., Guillot, S., Godard, M., Debret, B., and Nicollet, C., 2013, Highpressure serpentinites, a trap-and-release system controlled by metamorphic conditions: example from the Piedmont zone of the western Alps: Chemical Geology, v. 343, p. 38-54.

Mével, C., Caby, R. and Kienast, J.R., 1978, Amphibolite facies conditions in oceanic crust: example of amphibolitized flaser gabbros and amphibolites from the Chenaillet ophiolite massif (Hautes Alpes, France): Earth and Planetary Science Letters, v. 39, p. 98-108.

Millet, M.A. Baker, J.A., and Payne, C.E., 2012, Ultra-precise stable Fe isotope measurements by high resolution multiple-collector inductively coupled plasma mass spectrometry with a $57 \mathrm{Fe}-58 \mathrm{Fe}$ double spike: Chemical Geology, v. 304, p. 18-25.

Ohmoto, H, 1986, Stable isotope geochemistry of ore deposits in J.W Valley (Ed.), et al., Stable Isotopes in High Temperature Geological Processes: Reviews in Mineralogy, v. 16, p. 491-559

Paulick, H., Bach, W., Godard, M., De Hoog, J.C.M., Suhr, G. and Harvey, J., 2006, Geochemistry of abyssal peridotites (Mid-Atlantic Ridge, $15^{\circ} 20^{\prime} \mathrm{N}$, ODP Leg 209): implications for fluid/rock interaction in slow spreading environments. Chemical Geology, v. 234, p. 179-210. 
Pelletier, L. and Müntener, O., 2006, High-pressure metamorphism of the Lanzo peridotite and its oceanic cover, and some consequences for the Sezia-Lanzo zone (northwestern Italian Alps): Lithos, v. 90, p. 111-130.

Rouméjon, S., Cannat, M., Agrinier, P., Godard, M., and Andreani, M. Serpentinization and Fluid Pathways in Tectonically Exhumed Peridotites from the Southwest Indian Ridge (62-65 ${ }^{\circ}$ ): Journal of Petrology, doi:10.1093/petrology/egv014.

Schwartz, S., Allemand, P. and Guillot, S., 2001, Numerical model of the effect of ser- pentinites on the exhumation of eclogitic rocks: insights from the Monviso ophiolitic massif (Western Alps): Tectonophysics, v. 342, p. 193-206.

Schwartz, S., Guillot, S., Reynard, B., Lafay, R., Debret, B., Nicollet, C., Lanari, P. and Auzende, A.L., 2013, Pressure-temperature estimates of the lizardite/antigorite transition in high pressure serpentinites, Lithos, v. 178, p. 197-210.

Tricart, P. and Schwartz, S., 2006, A north-south section across the Queyras Schistes lustrés (Piedmont zone, Western Alps): syncollision refolding of a subduction wedge: Eclogae Geologicae Helvetiae, v. 99, p. 429-442.

Vitale Brovarone, A., Beyssac, O., Malavieille, J., Molli, G., Beltrando, M. and Compagnoni, R., 2013, Stacking and metamorphism of continuous segments of subducted lithosphere in a high-pressure wedge: the example of Alpine Corsica (France): Earth-Science Review, v. 116, 35-56.

Weyer, S., and Ionov, D.A., 2007, Partial melting and melt percolation in the mantle: the message from Fe isotopes. Earth and Planetary Science Letters, v. 259, p. 119-133.

Williams, H.M., Peslier, A.H., McCammon, C., Halliday, A.N., Levasseur, S., Teutsch, N., and Burg, J.P., 2005, Systematic iron isotope variations in mantle rocks and minerals: the effects of partial melting and oxygen fugacity. Earth and Planetary Science Letters, v. 235, p. 435-452.

Williams, H.M., and Bizimis, M., 2014. Iron isotope tracing of mantle heterogeneity within the source regions of oceanic basalts. Earth and Planetary Science Letters, v. 404, p. 396-407. 